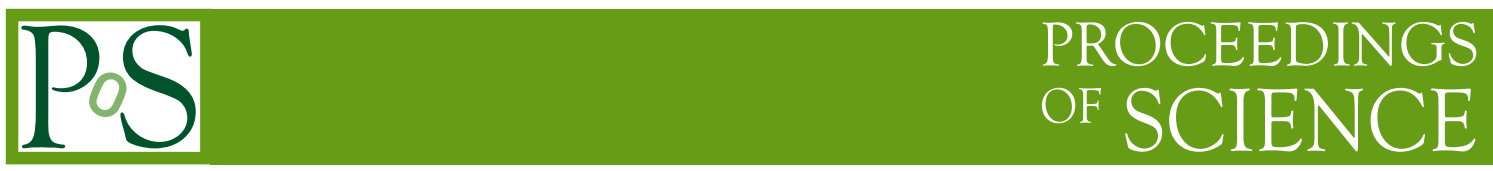

\title{
High School Students' Cosmic Ray Measurements During a Total Solar Eclipse
}

36th International Cosmic Ray Conference -ICRC2019-

July 24th - August 1st, 2019

Madison, WI, U.S.A.

\section{Anthony Valsamis ${ }^{\dagger}$}

Glenbrook North High School

Science Department, 2300 Shermer Road, Northbrook, IL 60062, USA

E-mail: avalsamiseglenbrook225.org

\section{Clarissa Carr, Jacob Rosenberg, Nathan A. Unterman}

Glenbrook North High School

Science Department, 2300 Shermer Road, Northbrook, IL 60062, USA

E-mail: nuntermanegmail. com

\section{Tamar Dallal, Michelle Matten, Jacob Miller, Ezra Schur, Allen Sears}

Ida Crown Jewish Academy

Science Department, 8233 North Central Park Avenue, Skokie, IL 60076, USA

E-mail: asearseicja.org

\section{Mark Adams}

University of Illinois at Chicago

Physics Department, 845 West Taylor Street, Chicago, IL 60607, USA

E-mail: adams@uic.edu

\begin{abstract}
:
Many high schools use QuarkNet cosmic ray muon detectors to learn the scientific process through a variety of experiments investigating cosmic rays. The total solar eclipse of August 21, 2017 provided an opportunity to measure any deviation in secondary cosmic ray flux more than $2 \mathrm{GeV}$. Students and teachers from high schools near Chicago designed telescopes to carry out measurements of the rate of cosmic ray muons in the direction of the sun during the August 21, 2017 North American solar eclipse. As an independent study project, teams constructed prototypes, measured background rates of muons, and designed two types of telescopes. For four days they operated four types of telescopes in a location where the eclipse was total. It was hypothesized that during a total solar eclipse, the muon flux changes. This research was possible due to an outreach program from QuarkNet, funded by the National Science Foundation.
\end{abstract}

${ }^{\dagger}$ Speaker 


\section{Feasibility and Design}

To measure the change in muon-coincident events during a total solar eclipse, a feasibility study was conducted to evaluate if the four scintillator counters could detect an event occurring with a small angle of acceptance. An overlapping design was chosen to be able to measure angles of acceptance both on-axis and off-axis.

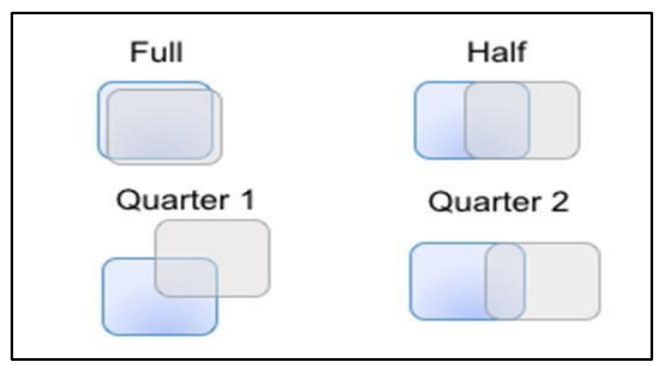

Figure 1 Overlap designs tested. Quarter 1 was selected for the experiment design.

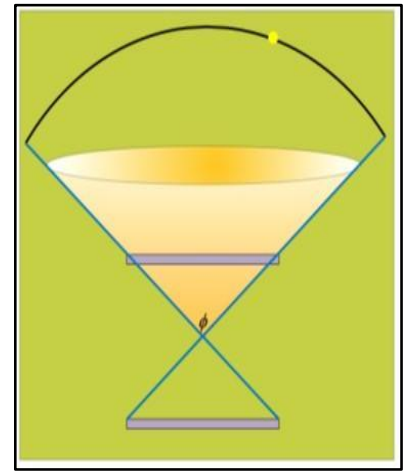

Figure 2 Angle of Acceptance

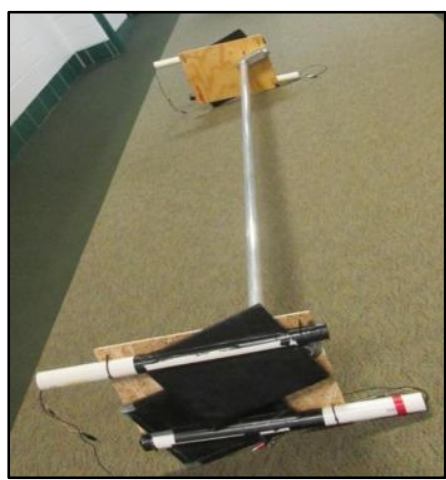

Figure 3 Telescope Design

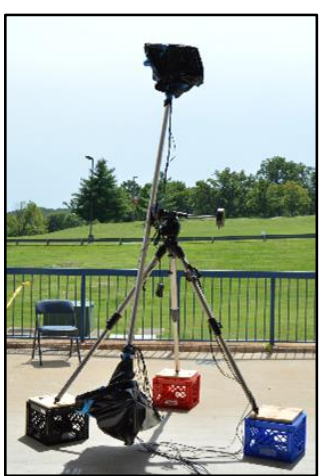

Figure 4 Assembled tracking telescope

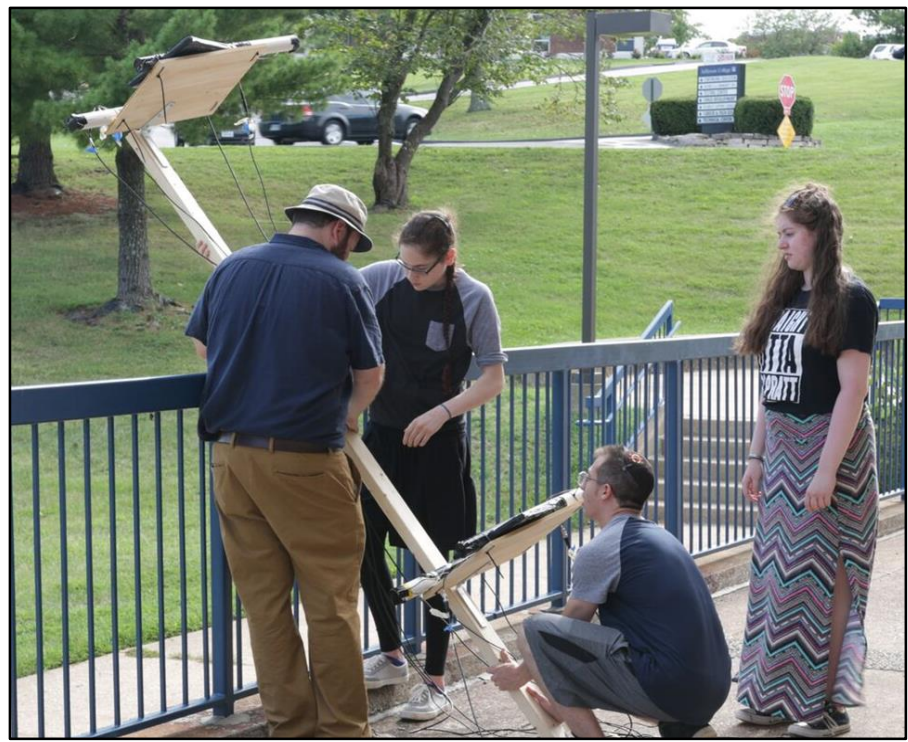

Figure 5 Students and teacher aligning the fixed telescope. 
In the days leading up to the eclipse, events were measured by telescopes that tracked the sun, moon, and empty sky. The fixed telescope also recorded events during a transit of the sun and moon as well as empty sky.

\section{Pressure, Temperature, and Normalization}

The data trend of the barometric pressure throughout the experiment demonstrates a change of $\pm 0.3 \%$. The temperature also remained relatively constant and normalization against the stacked control counters yielded negligible change in data trends. By binning the data in 10minute intervals, variations were minimal.

Counts occurring within the same detector with different angles of acceptance, called N, controlled the data for external factors. Additionally, counts taken from the control stacked array, called N', normalized counts for environmental changes that affect flux including changes in temperature and pressure. Pre-eclipse studies of solar and lunar transits indicate that the celestial bodies entering the detector's cone of acceptance yield no significant change in the number of events.

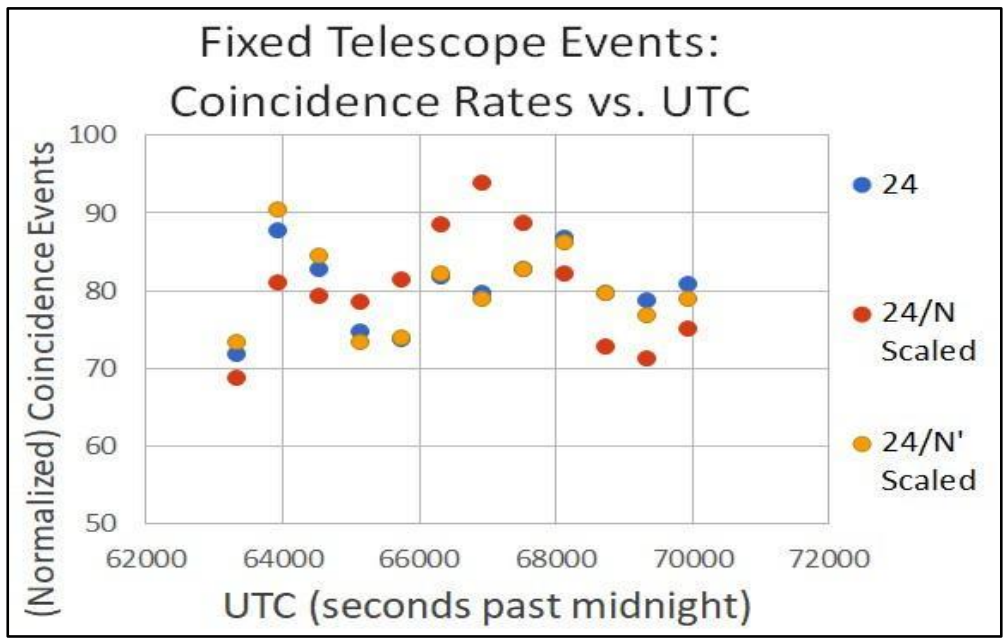

Figure 6 Fixed telescope results for a solar transit showing events per 10 minutes.

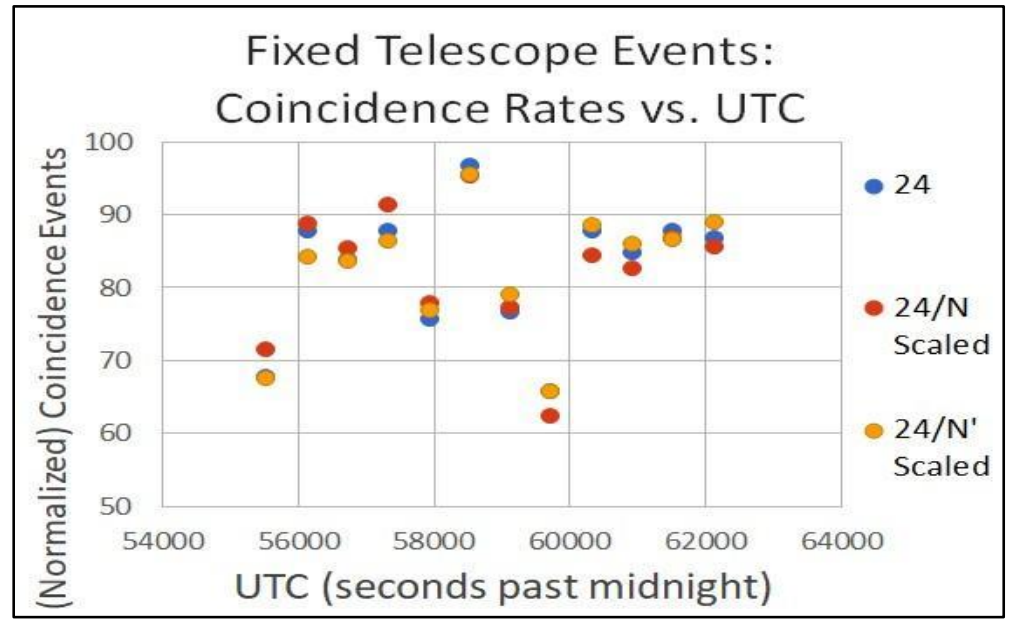

Figure 7 Fixed telescope results for a lunar transit showing events per 10 minutes.

Blank sky measurements also showed no significant change in the number of events. 


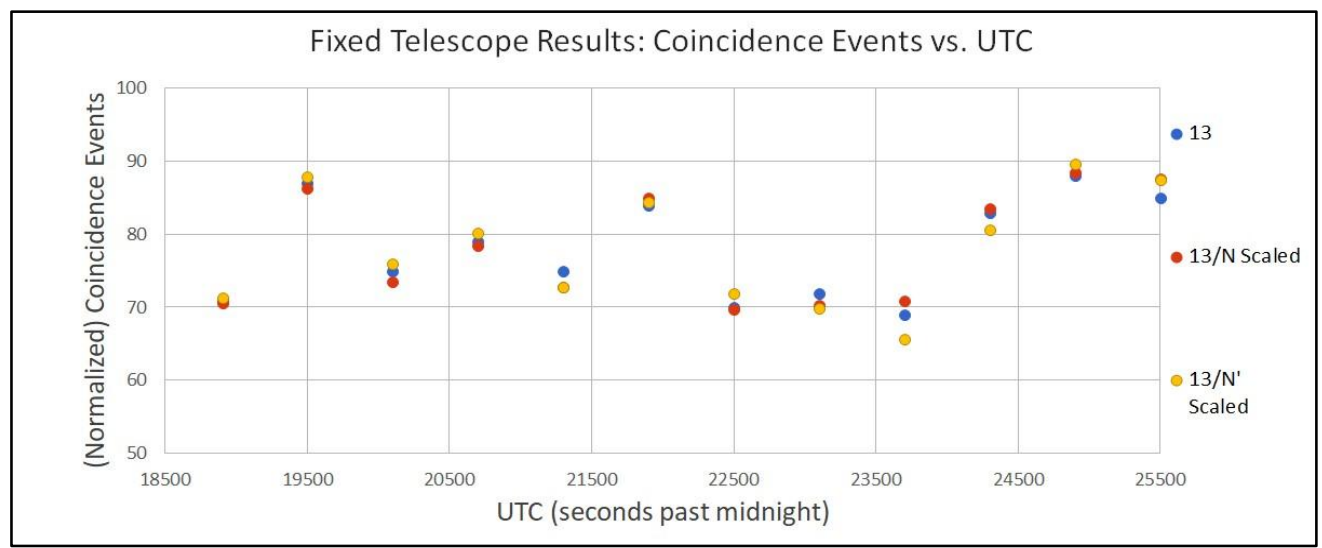

Figure 8 Fixed telescope results for blank sky for events per 10 minutes.

\section{Findings}

Data taken during the eclipse revealed no measurable divergence from the baseline.

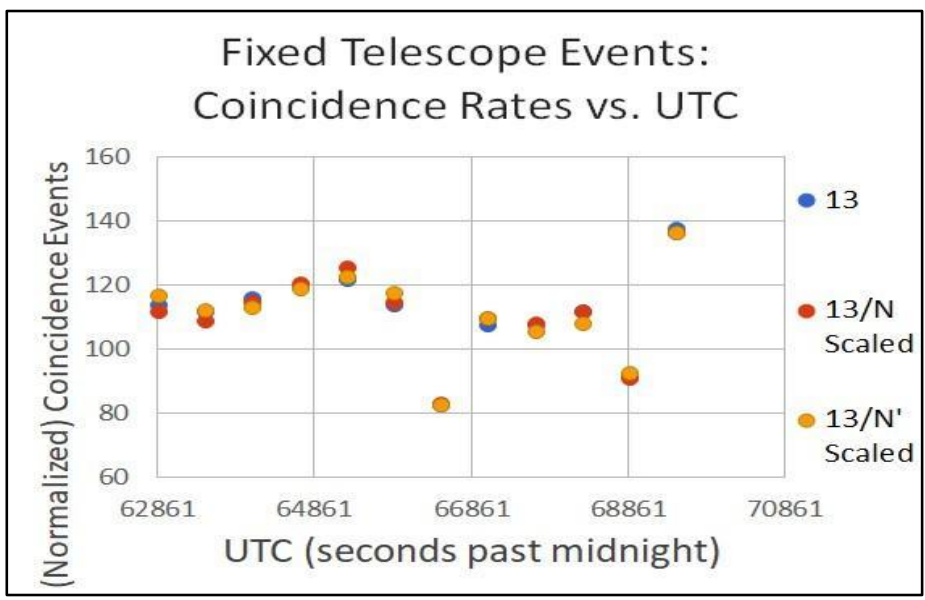

Figure 9 Fixed telescope results for eclipse for events per 10 minutes.

The telescopes that tracked the sun throughout the eclipse, events were normalized for the angle of solar elevation also remained constant for both the north-south and east-west axes, even as the moon entered and exited the detector's cone of acceptance, no signal was observed. 


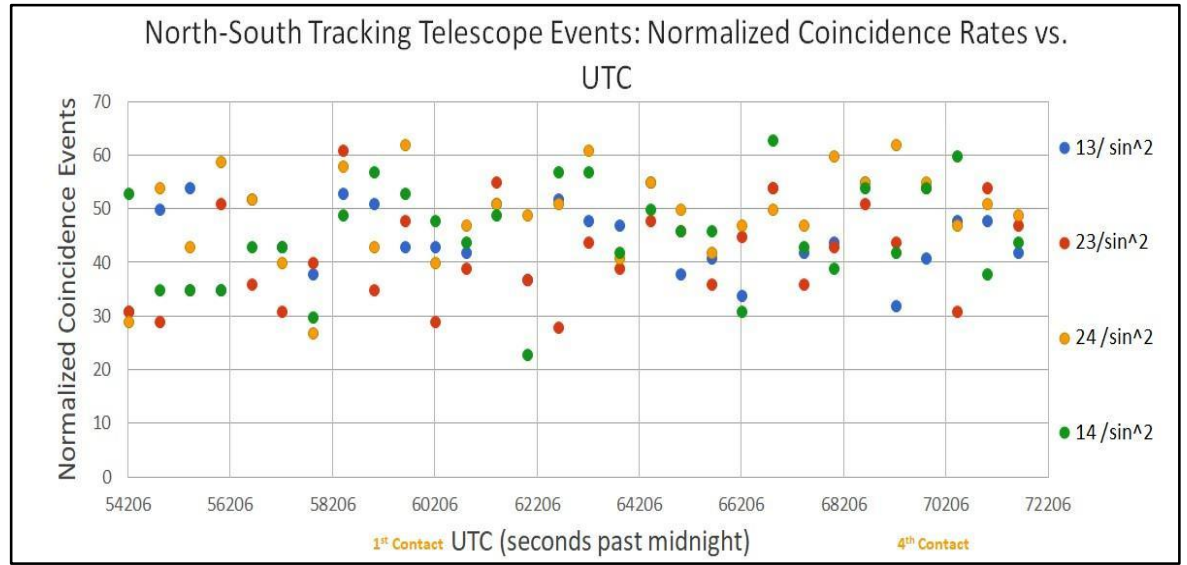

Figure 10 North-south tracking telescope results of normalized events per 10 minutes.

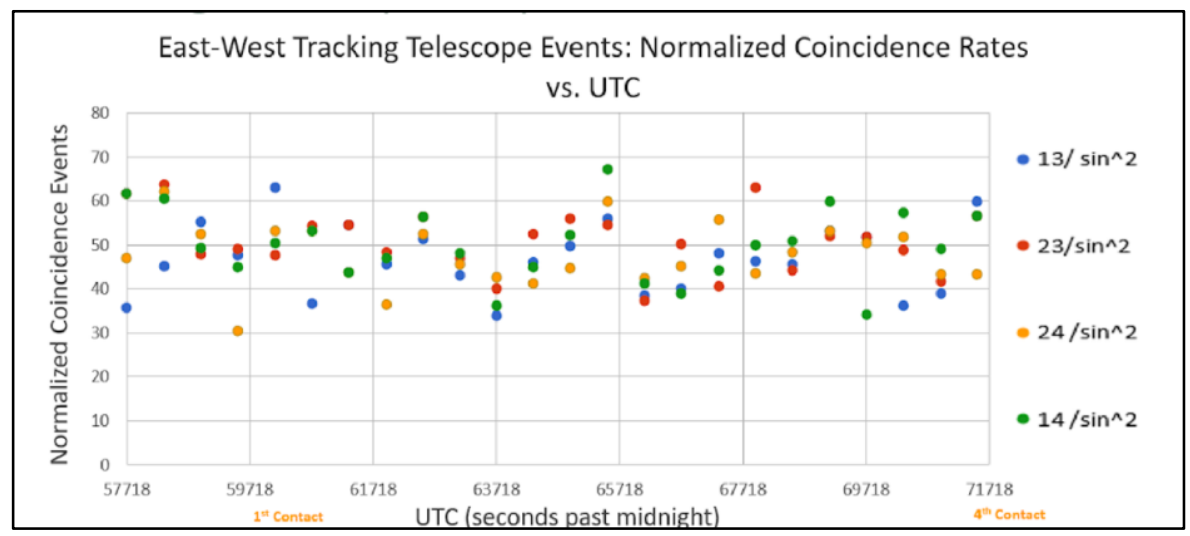

Figure 11 East-West tracking telescope in events per 10 minutes.

The following is a display of binning effects and normalization effects on the data. Notice how the data is proportional to the $\sin ^{2} \theta$ of the angle of elevation.

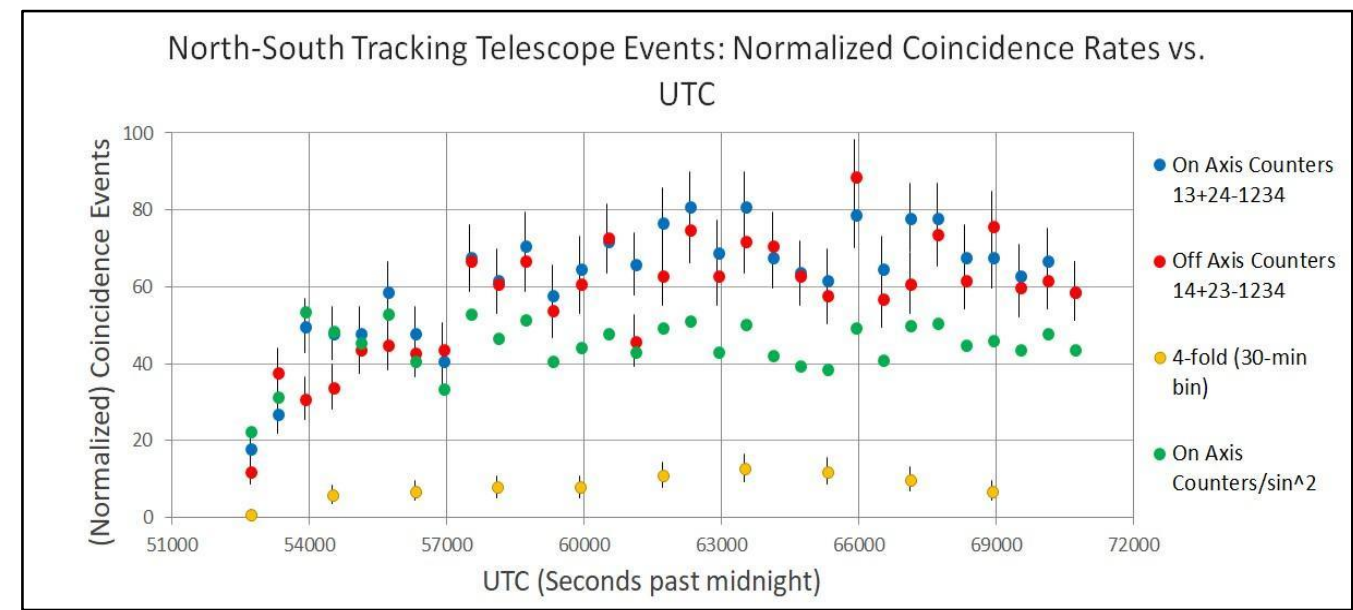

Figure 12 Eclipse results depicting binning effects, normalization effects, and differences between on-axis vs. off-axis data. 
The normalization illustrates the eclipse resulted in no significant change in the number of events within the limits of our equipment. The yellow data measured in 30 minute bins, as opposed to the 10 minute bins used throughout the study, reaffirm that larger bin size yields no change in results.

\section{Conclusion}

Results from this study show no meaningful change in cosmic events during the eclipse. The sun and moon are neither sources nor blockers of cosmic ray muons with energies $2 \mathrm{GeV}$ and higher. It is curious how large celestial objects such as the sun and moon did not obstruct muons significantly.

This experiment could be further improved and investigated by considering bin sizes, number of detectors, location of detectors, and the portion of the sky being examined. By analyzing the data in larger bin sizes, the data will be more statistically significant at the cost of the ability to discern a more precise timed signal related to the eclipse. Decreasing bin sizes results in increased precision at the cost of statistical significance. By using and analyzing data from more detectors in more additional locations, statistical significance would be further increased and the effects of Earth's magnetic field could be explored.

We look forward to testing some of this these possibilities in 2024! We hope that the outreach support of QuarkNet continues for high energy physics education at the secondary level.

\section{Acknowledgements}

Eclipse Day Host: Robert Brazzle, Jefferson College, Hillsboro, MO

Science Departments and administration of Glenbrook District 225 High Schools and Ida Crown Jewish Academy.

QuarkNet 